\title{
The similarities between Long-haul COVID-19 and myalgic encephalomyelitis/ /chronic fatigue syndrome (ME/CFS)
}

Key words: ME/CFS, Long-haul COVID-19, fatigue

\section{To the Editor:}

A large number of research studies are currently collecting data about the whole range of short- and long-term health effects associated with SARS-CoV-2 infection. Long COVID-19, post-COVID-19 also known as Long-haul COVID-19 is the current name being given to the long-term sequelae (persistent symptoms experienced longer than 6 weeks) of SARS-CoV-2 infection. An estimated $10 \%$ of patients with COVID develop Long-haul COVID-19 symptoms [1]. Initial reports suggest that post viral fatigue (PVF) and Post viral fatigue syndrome (PVFS) are the most common long term issues in individuals infected with SARS-CoV-2. The severity and duration of the acute viral infection are strong risk factors for the development of fatigue syndromes. According to an Australian research group, approximately $12 \%$ of 253 subjects developed a Post Viral Syndrome that involved fatigue, cognitive dysfunction, mood disturbance and musculoskeletal pain [2].

Postinfectious syndromes including those seen with persistent symptoms after COVID-19, often share a common symptom phenotype, that is either self-limiting or has features that are very similar to myalgic encephalomyelitis/chronic fatigue syndrome (ME/CFS) [1, 3]. Recently, there have also been reports about a high frequency of patient-reported Longhaul-COVID-19 symptoms that as well as fatigue include cognitive difficulties (problems with short term/working memory, concentration, information processing), pain, orthostatic intolerance (problems in remaining upright, feeling dizzy), anxiety/depression, sleep and autonomic disturbances [4]. One symptom that is considered to be unique to ME/CFS is post-exertional malaise (PEM). PEM can be described as delayed and significant exacerbation of ME/CFS symptoms that always follow physical and cognitive activity [1].

Although ME/CFS and Long-haul COVID-19 are medically distinct, they share a common pathological process including viral infection, altered mitochondrial dynamics with sequent oxidative stress, pro-inflammatory state, cytokine production, and cell death [3]. Acute Epstein-Barr virus (EBV) infection is an important virus trigger of ME/CFS. Some researchers speculate that SARS-CoV-2 could replace the EBV as being the most frequent precipitating event for ME/CFS or Long-haul COVID-19. Considering the overlapping of symptoms, ME/CFS and Long-haul COVID-19 should be better characterized as post active phase of infection syndromes (PAPIS) [1]. Furthermore, the opportunities to learn about pathophysiology and treatments from the study of these commonalities and differences in each disease should be recognised.

PVFS may also result from immune system overreaction associated with ongoing production of increased cytokine levels, especially interleukins 6 and 10. These cytokines are also thought to play a key role in immune dysregulation and are frequently reported as abnormal in many cases of ME/CFS. In patients with Longhaul-19 symptoms lasting for more than 3 months with a functional impairment that prevents a return to everyday activities (education, employment) a diagnosis of ME/CFS should be considered. [2-3]. Medicum in Bydgoszcz Nicolaus, Copernicus University in Torun, Poland, e-mail: p.zalewski@cm.umk.pl 
Patient evidence also indicates that a good initial approach to the management of PVS reduces the probability of this turning into ME/CFS. The standard approach to the management of any form of significant post viral fatigue involves convalescence and a gradual return to normal daily activities. A recent study using an MRI scan showed cardiac inflammatory involvement in both hospitalised and non-hospitalised subgroups. Thus, proper evaluation of cardiac and respiratory function is needed [2]

This is an important time for those currently with, or at risk of postinfectious syndromes which includes some with ME/CFS. Exploration of the causation and effective treatment of such syndromes will improve the understanding and treatment regardless of the triggering illness. Novel research into COVID-19 may lead to a much better understanding of the role of the immune response and why some people develop post viral syndromes and ME/CFS [1, 4].

\section{References}

1. Friedman KJ, Murovska M, Pheby DFH, et al. Our Evolving Understanding of ME/CFS. Medicina (Kaunas). 2021; 57(3), doi: 10.3390/medicina57030200, indexed in Pubmed: 33652622.

2. Shepherd C. Post COVID-19 Fatigue, Post/Long Covid-19 Syndromes and Post-Covid ME/CFS. www.meassociation.org.uk (September 2020).

3. Gaber T. Assessment and management of post COVID fatigue. Progress in Neurology and Psychiatry. 2021; 25(1): 36-39, doi: 10.1002/pnp.698

4. Aucott JN, Rebman AW. Long-haul COVID: heed the lessons from other infection-triggered illnesses. Lancet. 2021; 397(10278): 967-968, doi: 10.1016/S0140-6736(21)00446-3, indexed in Pubmed: 33684352. 